\title{
Design of electromagnetic band gap media based on crank-like chiral structures
}

\author{
Ángel J. García-Collado Member, IEEE, Gregorio J. Molina-Cuberos, Óscar Fernández, \\ and José Margineda Member, IEEE
}

\begin{abstract}
A new EBG (Electromagnetic Band Gap) structure with chiral crank elements is presented and its effects on the electric field propagation along a microstrip line are analysed and compared with the effects produced by mushroom-type inclusions. Chiral crank inclusions are distributed along both sides of the strip and the corresponding results are compared with those obtained by other elements commonly used to create EBG media, mushroom-type structures. A single row of high chirality structures on both sides of a transmission line produces a band gap with a wider bandwidth than that obtained by the inclusion of other elements commonly used to create EBG media, such as mushroom-type structures. Unlike what happen with mushroomtype inclusions, more rows of chiral elements do not change significantly the central frequency and bandwidth of the band gap. The proposed structure works in X-Band (8.2-12.4 GHz) and it is easily adaptable to other frequencies by changing the size of the crank inclusions. Several samples have been made in FR-4 printed circuit board and a good agreement has been found between numerical analysis and experimental measurements.
\end{abstract}

Index Terms-Electromagnetic metamaterials, chiral media, microstrip line, microwave filters.

\section{INTRODUCCIÓN}

Durante las últimas décadas, se han logrado avances muy importantes en el desarrollo de nuevos materiales artificiales con propiedades electromagnéticas de gran interés. Estos medios abren la puerta de nuevas aplicaciones relacionadas con la propagación de ondas, antenas, dispositivos pasivos de microondas, superficies de alta impedancia, etc.

Los medios EBG (Electromagnetic Band Gap) son estructuras artificiales, normalmente periódicas, que permiten la propagación de ondas electromagnéticas únicamente en una banda de frecuencias estrecha, independientemente del ángulo de incidencia o polarización [1], [2]. Además, evitan la propagación de ondas superficiales y mejoran las características de propagación de las ondas [3], la radiación en antenas [4], [5], o en aplicaciones de telefonía móvil [6].

Una de las estructuras más estudiadas para diseñar medios EBG es la conocida como de tipo hongo, del término

Submitted on March 16, 2020. This work has been supported by the Ministerio de Economía y Competitividad of Spain (ERDF co-funded Research Projects PGC2018-098350-B-C21 and PGC2018-098350-B-C22.

A. J. García-Collado (ajgarcia@ucam.edu) is with Dpto. Ciencias Politécnicas. Grupo de Teledetección, Geomática y Sistemas de Información Geográfica Aplicados, Universidad Católica de Murcia, E-30107 Murcia, Spain.

G. J. Molina-Cuberos (gregomc@um.es) and J. Margineda (jmargi@um.es) are with Dpto. de Electromagnetismo y Electrónica, Universidad de Murcia, Campus de Espinardo, E-30100 Murcia, Spain.

O. Fernández (oscar.fernandez@unican.es) is with Dpto. de Ingeniería de Comunicaciones, Universidad de Cantabria, E-39005 Santander, Spain.

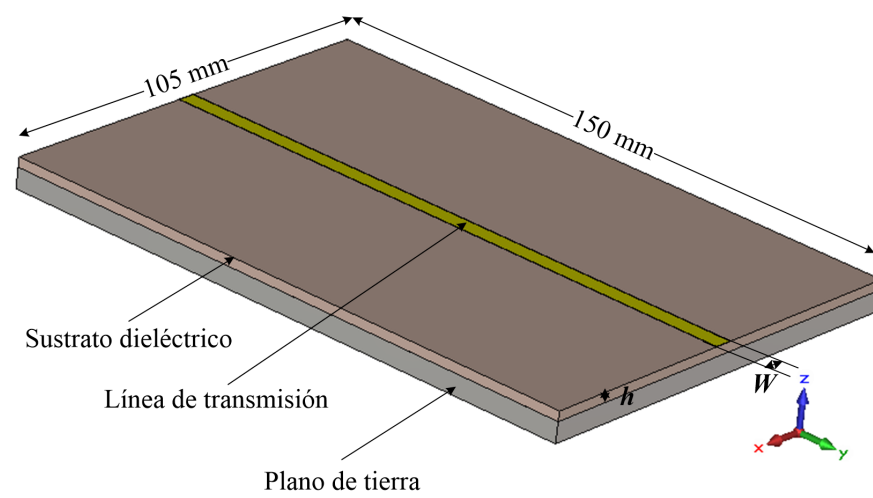

Fig. 1. Línea microstrip de impedancia característica $50 \Omega$.

anglosajón mushroom-type, desarrollado por Sievenpiper et al. [7]. En este trabajo adoptaremos la denominación mushroom por ser un término ampliamente empleado y conocido en el campo de conocimiento de los metamateriales. Esta estructura posee ciertas ventajas, como su forma compacta, se puede integrar fácilmente usando tecnología de circuito impreso $(P C B)$ proporcionando estructuras de bajo coste y se puede adaptar fácilmente en frecuencia cambiando las dimensiones del mushroom. Sin embargo, su respuesta se limita a bandas de frecuencias estrechas, lo que reduce su utilización en aplicaciones que requieren banda ancha de frecuencias [8][10].

En este trabajo presentamos un nuevo medio EBG basado en la inserción de estructuras quirales en forma de manivela (crank). Analizamos el efecto producido en una línea microstrip cuando se añaden estructuras de este tipo a ambos lados de la misma y lo comparamos con el efecto producido cuando se utilizan estructuras de tipo mushroom. Utilizaremos una herramienta de simulación numérica para obtener los parámetros $\mathrm{S}$ de las estructuras y compararemos los resultados obtenidos con medidas experimentales.

\section{Planteamiento y DESCRIPCión DE LAS MUESTRAS}

Analizaremos y compararemos la respuesta de una línea microstrip estándar con líneas microstrip con una o más filas de estructuras tipo mushroom a ambos lados de la línea. Los parámetros $\mathrm{S}$ se determinan simulando el conjunto con CST Microwave Studio ${ }^{T M}$, prestando una atención especial al parámetro de transmisión con el objetivo de estudiar la banda de transmisión prohibida. Por conveniencia con nuestro sistema experimental, trabajamos en banda-X (8.2 a 12.4 $\mathrm{GHz}$ ), aunque los resultados son fácilmente extensibles a 
otras bandas modificando a decuadamente 1 as dimensiones de las estructuras. Seguiremos la norma usual para definir la banda prohibida de la estructura EBG como el intervalo de frecuencias en el que el parámetro $S_{21}$ disminuye más de $10 \mathrm{~dB}$.

La línea microstrip se fabrica en cobre sobre un sustrato estándar de FR-4 $\left(\epsilon_{r}=4.3\right.$ a $\left.10 \mathrm{GHz}\right)$ de dimensiones $105 \mathrm{~mm} \times 150 \mathrm{~mm}$ y $2.4 \mathrm{~mm}$ de grosor $(h)$. La línea tiene una anchura $W=4.75 \mathrm{~mm}$, siendo su impedancia característica $Z_{0}=50 \Omega$ [11], y un grosor de cobre de $35 \mu \mathrm{m}$ (Fig. 1). La elección de la longitud de la línea de transmisión se debe fundamentalmente a una adecuación al sistema de medida empleado en el proceso experimental. Se ha comprobado que con una longitud inferior (3 ó $4 \mathrm{~cm}$, con la consiguiente reducción en el número de inclusiones laterales que ello implica) los resultados son equivalentes.

Siguiendo la propuesta inicial de Sievenpiper et al. [7], se añaden una o más filas de mushrooms a cada lado de la línea microstrip (Fig. 2). El parche cuadrado que forma el mushroom tiene un lado de $D_{m}=6.5 \mathrm{~mm}$ y usamos una distancia de separación entre cuadrados $g=0.5 \mathrm{~mm}$. La longitud de las vías metálicas que conectan el parche con el plano de tierra es de $0.24 \mathrm{~mm}$, altura que coincide con el grosor del sustrato dieléctrico $h=2.4 \mathrm{~mm}$. El grosor del parche metálico es el mismo que el de la línea de transmisión. La distancia entre el borde de la línea de transmisión y los parches es de $1.60 \mathrm{~mm}$. Todas estas dimensiones han sido calculadas para observar los efectos significativos en la banda-X y, de este modo, poder ser contrastados experimentalmente. La implementación de los prototipos se llevó a cabo siguiendo una técnica de revelado PCB manual. Las medidas de los parámetros $\mathrm{S}$ se realizaron utilizando un instrumento de medida Agilent PNA-L Network Analyzer (N5230A).

A continuación, una o más filas d e c eldas c on cuatro manivelas se insertan a ambos lados de la línea microstrip, (Fig. 3). Las manivelas, ver detalle en Fig. 3, son estructuras tridimensionales que presentan comportamiento quiral [12]-[14], con las siguientes dimensiones: $D_{c}=5 \mathrm{~mm}$, $D_{c^{\prime}}=8 \mathrm{~mm}, l_{1}=l_{3}=3.5 \mathrm{~mm}, l_{2}=2.4 \mathrm{~mm}$, y anchura de segmento de $0.6 \mathrm{~mm}$. La vía (diámetro de $0.25 \mathrm{~mm}$ ) enlaza los segmentos superiores e inferiores atravesando el sustrato. Se colocan cuatro manivelas constituyendo una celda en forma de cubo para maximizar el parámetro de quiralidad. Al conjunto formado por cuatro manivelas lo denominamos 4CR (4 Crank Resonator). Al igual que con los mushrooms, las dimensiones se han optimizado para conseguir efectos en la banda de frecuencias de interés. En general, la longitud total de los segmentos que constituyen la manivela y la proximidad entre las manivelas colindantes modifican $\mathrm{la} b$ anda $\mathrm{d}$ e frecuencias en la que se producen los efectos observados. Un aumento en la longitud de la manivela o un incremento de la distancia entre inclusiones 4CR implica efectos en frecuencias más bajas. Los segmentos metálicos cerca del plano de tierra han sido aislados convenientemente. Las implementaciones físicas de algunas de las estructuras planteadas son mostradas en la Fig. 4. La Fig. 4(a) presenta la línea de transmisión sin inclusiones laterales, mientras que la Fig. 4(b) es la misma línea de transmisión con una fila de mushroom a sus lados y

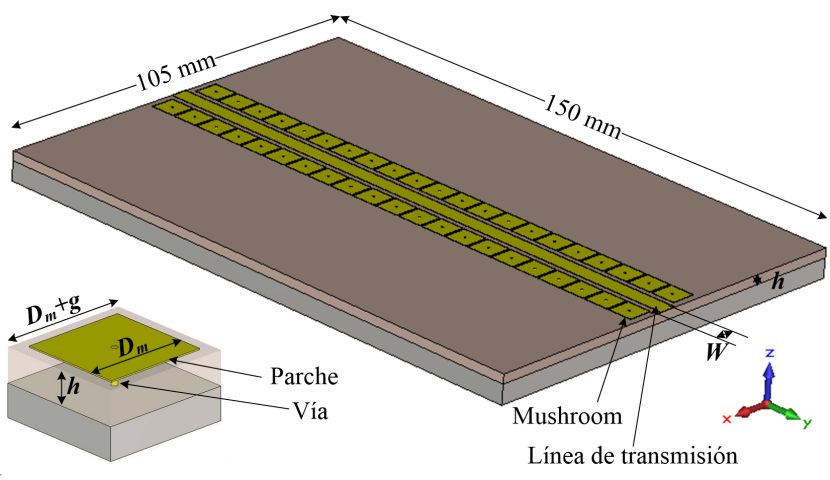

Fig. 2. Línea microstrip con una fila de mushrooms a cada lado y detalle del mushroom.

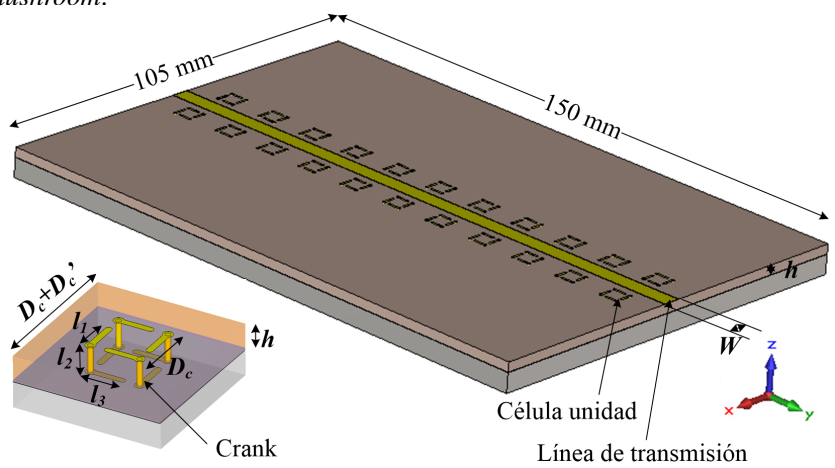

Fig. 3. Línea microstrip con una fila de celdas formadas por cuatro manivelas (4CR) a cada lado y detalle de la celda unidad de manivelas.
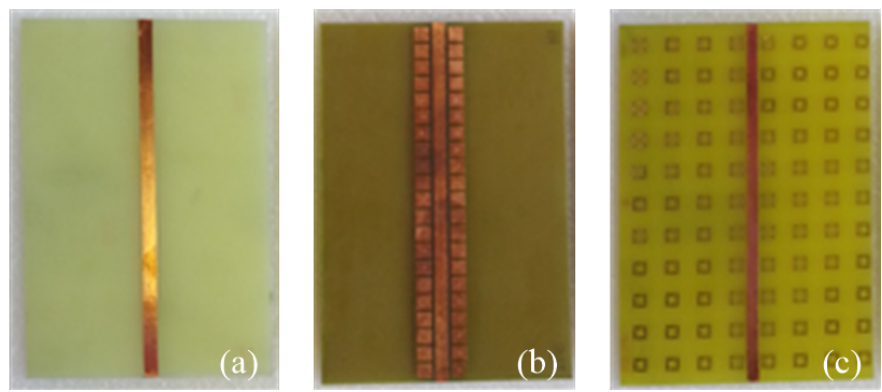

Fig. 4. Modelo implementado en sustrato FR-4: (a) línea de transmisión sin inclusiones, (b) línea de transmisión con inclusiones en forma de mushrooms y (c) línea de transmisión con inclusiones 4CR.

la Fig. 4(c) muestra la línea con cuatro filas de estructuras 4CR a ambos lados de la misma. La fabricación de los prototipos se realizó mediante un proceso industrial.

\section{RESULTADOS Y DISCUSIÓN}

La Fig. 5 muestra los resultados obtenidos para una línea de transmisión con una, dos y tres filas de mushrooms a cada lado de la línea. En la Fig. 5(a) se muestra los resultados obtenidos con la simulación numérica. El parámetro de transmisión $S_{21}$ de la línea sin inclusiones presenta una respuesta lineal en frecuencia, que se ve afectada únicamente por las pérdidas del sustrato dieléctrico. Podemos observar como la presencia de mushrooms a ambos lados de la línea produce una banda prohibida. Con una fila a ambos lados, la banda prohibida se extiende entre 10.7 hasta $11.7 \mathrm{GHz}$, lo que significa un ancho de banda de $1.0 \mathrm{GHz}$. Este ancho aumenta cuando 

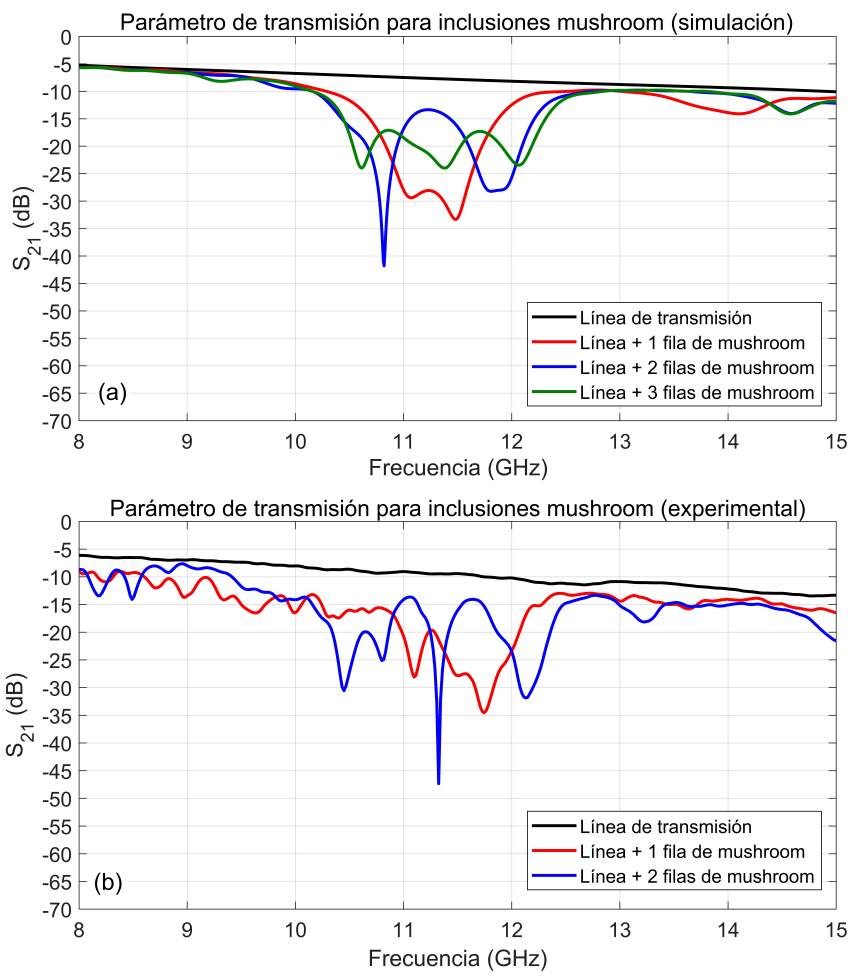

Fig. 5. (a) Simulación numérica. Parámetro de transmisión de la línea microstrip (negro), línea microstrip con una fila de mushrooms (rojo), con dos filas de mushrooms (azul) y con tres filas de mushrooms (verde). (b) Medidas experimentales. Igual para una y dos filas de mushrooms.

se incluyen más filas de mushrooms: $1.6 \mathrm{GHz}$ (entre 10.5 y $12.1 \mathrm{GHz}$ ) para dos filas y $1.7 \mathrm{GHz}$ (entre 10.5 y $12.2 \mathrm{GHz}$ ) para tres filas, si bien se ha de considerar la aparición de unos máximos relativos dentro de esas bandas, en especial en el caso de las dos filas. El número de mínimos en el parámetro de transmisión aumenta con el número filas, de forma que se obtiene un mínimo para una fila, dos mínimos para dos filas y tres mínimos para tres filas (siempre a ambos lados de la línea de transmisión). Cuanto mayor es el número de filas insertadas, mayor es el rizado obtenido.

La Fig. 5(b) muestra los resultados experimentales cuando se incluyen una y dos filas de mushrooms. Como puede observarse, hay un buen acuerdo con los resultados obtenidos en la simulación, con ciertas diferencias atribuibles fundamentalmente al proceso de fabricación, dando lugar a que las bandas prohibidas sean ligeramente mayores: $1.2 \mathrm{GHz}$ (entre $10.9 \mathrm{GHz}$ y $12.1 \mathrm{GHz}$ ) para una fila y $2.0 \mathrm{GHz}$ (entre $10.3 \mathrm{GHz}$ y $12.3 \mathrm{GHz}$ ) para dos filas. Los máximos relativos dentro de la banda prohibida también son observados en las medidas experimentales.

El parámetro $S_{21}$ de una línea de transmisión microstrip con 1, 2 y 4 filas de inclusiones de 4CR a ambos lados de la misma se presentan en la Fig. 6. Los resultados de la simulación, Fig. 6(a), muestran una banda prohibida de anchura similar para distinto número de filas, con un ancho de banda de aproximadamente $1.5 \mathrm{GHz}$ en todos los casos. La inclusión de más filas de este tipo de estructura no afecta significativamente al ancho de la banda prohibida, aunque sí se produce un aumento en las pérdidas por inserción. El rizado observado en la banda es similar en todos los casos
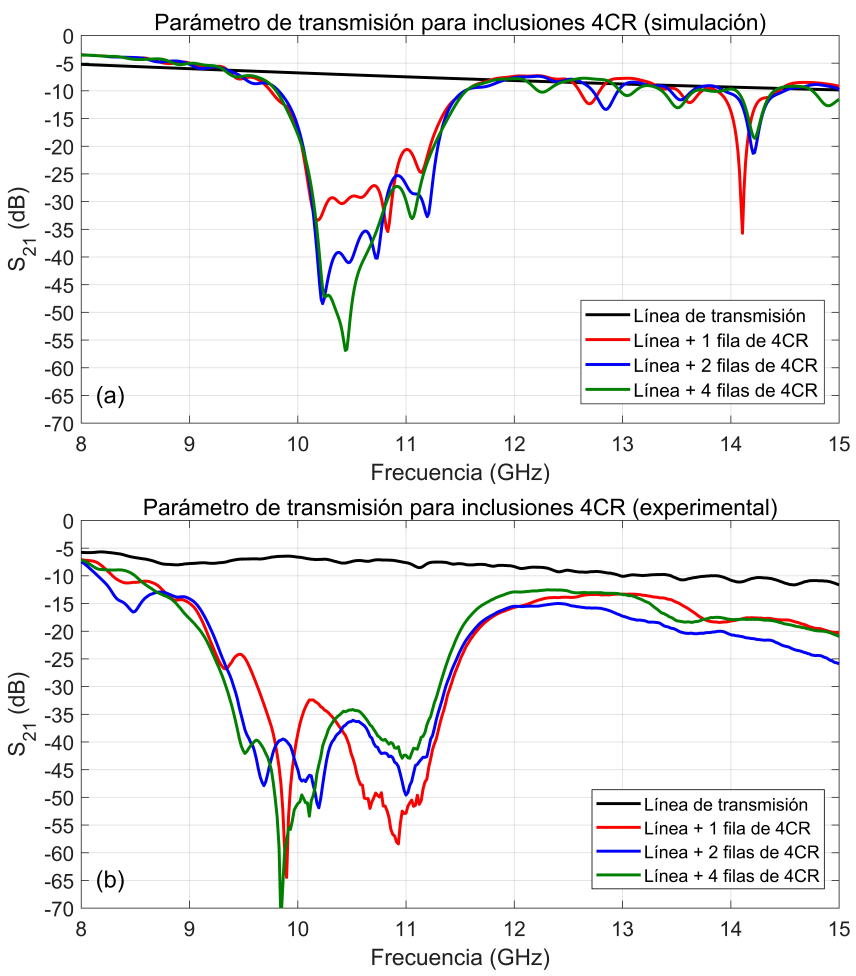

Fig. 6. (a) Simulación numérica. Parámetro de transmisión de la línea microstrip (negro), línea con una fila de inclusiones (rojo), con dos filas de inclusiones (azul) y con tres filas de inclusiones (verde). (b) Medidas experimentales. Igual para una y dos filas de inclusiones 4CR.

analizados y siempre inferior a $-20 \mathrm{~dB}$, a diferencia de las inserciones de mushrooms. Por tanto, los efectos observados son debidos, básicamente, a la primera fila de estructuras quirales y no es necesario incluir más filas para mejorar la respuesta. Los resultados experimentales, Fig. 6(b), corroboran este comportamiento, y se observa un aumento en la anchura de la banda prohibida $(\sim 3.2 \mathrm{GHz})$. Tanto simulaciones como medidas experimentales presentan una respuesta que no depende del número de filas de inclusiones metálicas. Se trata de un resultado lógico si se tiene en cuenta que la estructura resonante 4CR es más cerrada que la de tipo mushrooms, por lo que se produce una mayor concentración de los campos en la propia estructura, que se comporta casi como un resonador rectangular.

Si se modifica las dimensiones de los elementos quirales, la distancia entre ellos, o la distancia de éstos a la línea de transmisión, el parámetro $S_{21}$ también se verá afectado. En principio, es posible ajustar la localización de la banda prohibida y centrarla en otras frecuencias de interés. En general, un acercamiento de las filas a la línea de transmisión no afecta a la respuesta en frecuencia, pero si disminuye el valor del parámetro de transmisión. Además, una disminución de la distancia entre las filas de inserciones metálicas 4CR a lo largo del eje Y no afecta significativamente a los resultados aquí presentados.

Las Figuras 7 y 8 muestran los resultados presentados anteriormente solapando los datos experimentales y los datos de simulación numérica para las dos estructuras analizadas. Han sido incluidas a fin de poder observar el grado de similitud entre ambas medidas. 


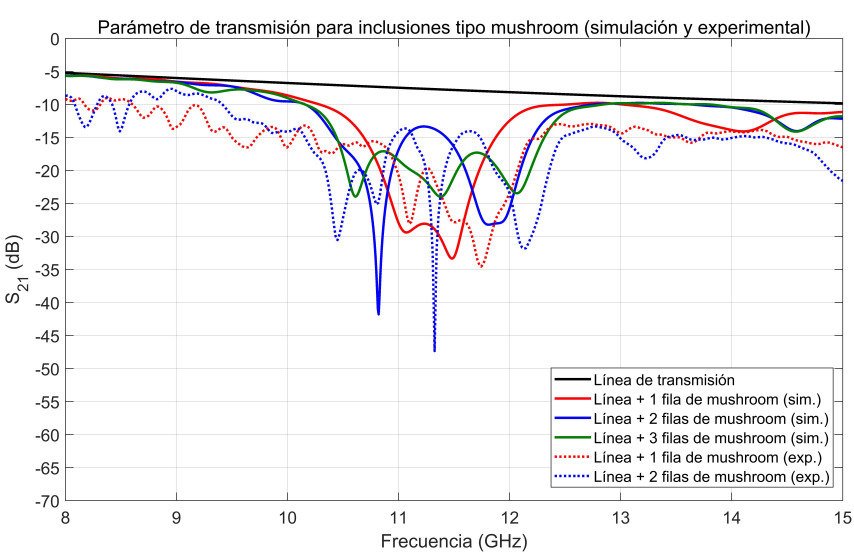

Fig. 7. Parámetro de transmisión para inclusiones de tipo mushroom (simulación -líneas continuas- y experimental -líneas de puntos-).

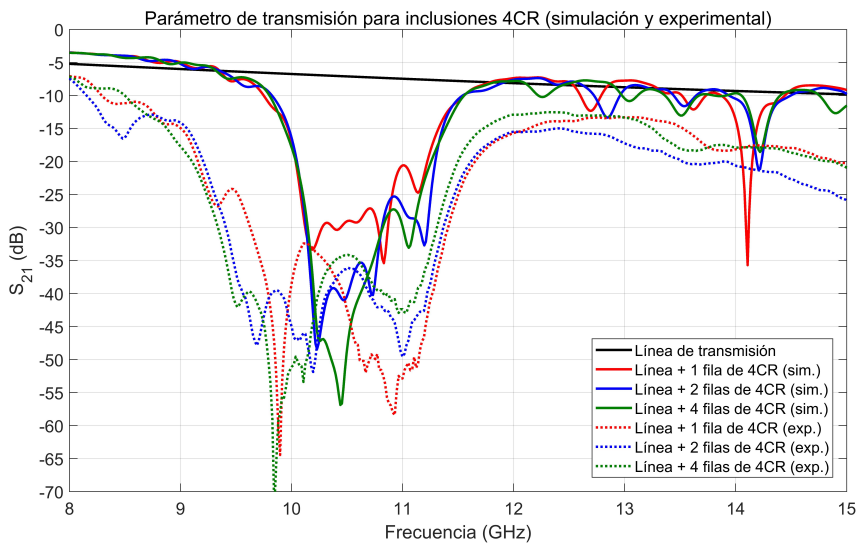

Fig. 8. Parámetro de transmisión para inclusiones de tipo 4CR (simulación -líneas continuas- y experimental -líneas de puntos-).
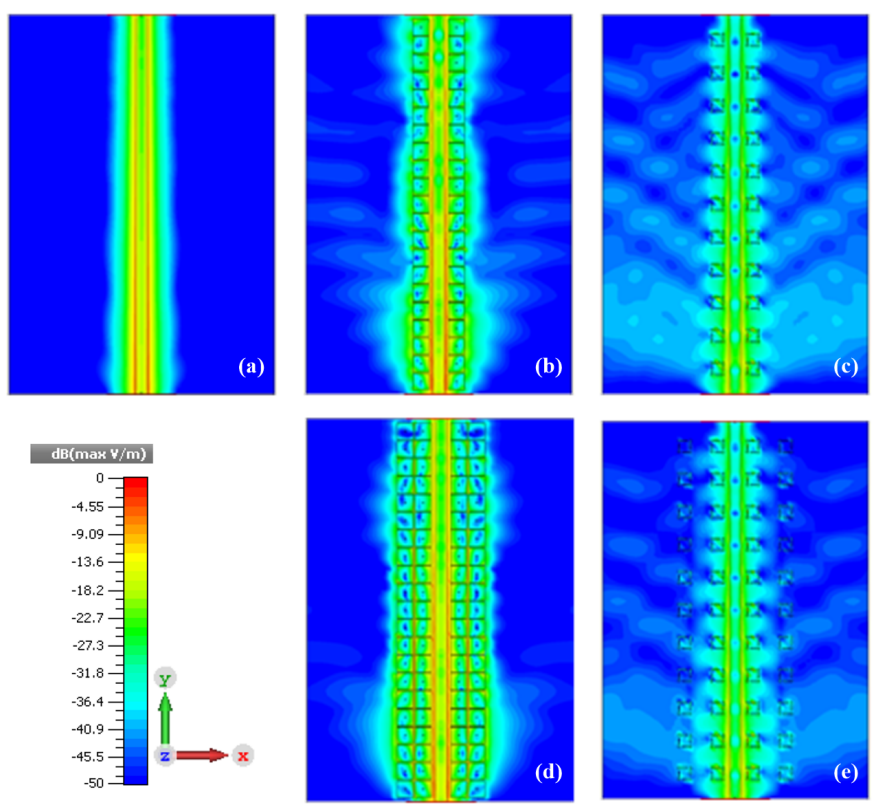

Fig. 9. Amplitud del campo eléctrico en la superficie del sustrato dieléctrico para una frecuencia de $9.5 \mathrm{GHz}$, fuera de la banda prohibida: (a) línea de transmisión, (b) línea de transmisión con una fila de mushrooms, (c) línea de transmisión con una fila de 4CR, (d) línea de transmisión con dos filas de mushrooms, (e) línea de transmisión con dos filas de 4CR. Todas las figuras con la misma escala.

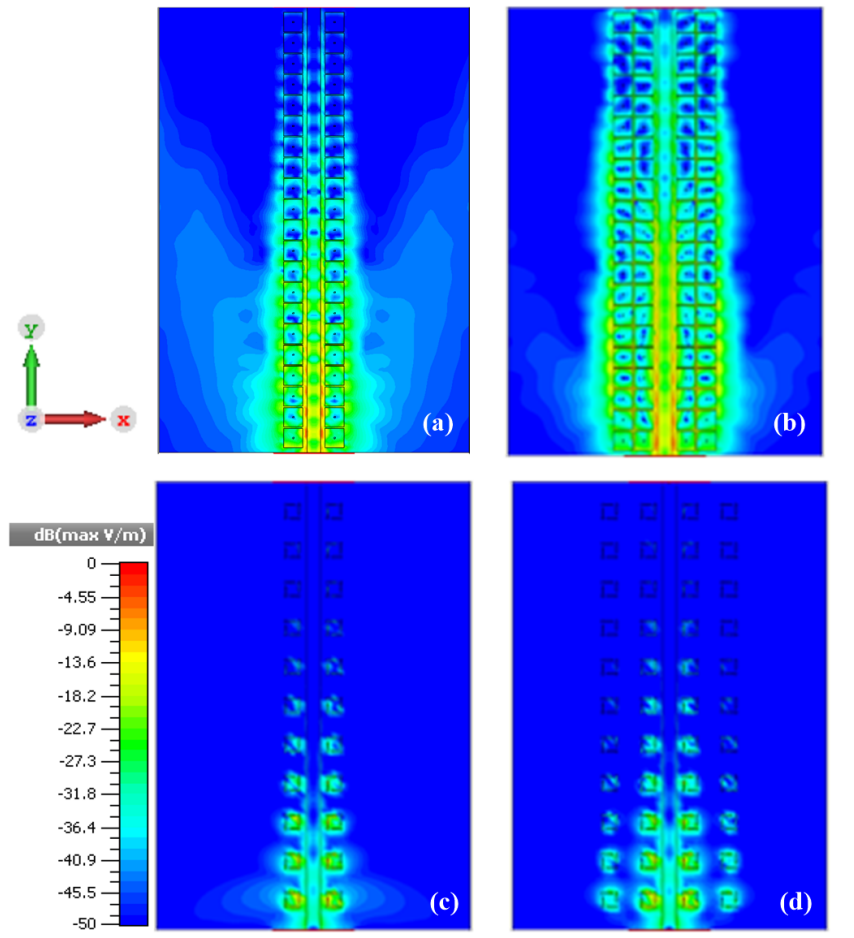

Fig. 10. Amplitud del campo eléctrico en la superficie del sustrato dieléctrico a diferentes frecuencias: (a) una fila de mushrooms $(11.5 \mathrm{GHz})$, (b) dos filas de mushrooms $(10.82 \mathrm{GHz})$, (c) una fila de 4CR (10.9 GHz), (d) dos filas de 4CR $(10.9 \mathrm{GHz})$. Todas las figuras con la misma escala.

La Fig. 9 muestra la amplitud del campo eléctrico en la superficie del dieléctrico en una frecuencia fuera de la banda prohibida, y la Fig. 10 en una frecuencia contenida en la banda prohibida. A fin de tener una referencia de partida, la Fig. 9(a) presenta la distribución de campo eléctrico en la línea de transmisión estándar. Tal y como era de esperar, la señal se transmite totalmente en la línea y el campo queda confinado cerca de ésta. Las Figs. 9(b) y 9(d) muestran la distribución de campo eléctrico en la línea con una y dos filas de mushrooms, respectivamente, mientras que las Figs. 9(c) y 9(e) presentan el campo en la línea con una y dos filas de inclusiones 4 CR. En todos los casos la señal se transmite por la línea y la amplitud de campo eléctrico aumenta en puntos alejados de la misma. En el caso de las inclusiones mushrooms, la zona con campo es mayor cuando hay dos filas. Ésto no ocurre para las inclusiones 4CR. En este caso la región donde están confinados los campos es prácticamente idéntica con una y dos filas de este tipo de elementos.

Las Figs. 10(a) y 10(b) presentan el comportamiento de la línea de transmisión con una y dos filas de mushrooms a una frecuencia contenida dentro de la banda prohibida. Se ha elegido la frecuencia donde el parámetro $S_{21}$ alcanza un mínimo. Se puede observar en ambos casos una atenuación en la señal con respecto a los valores mostrados en las Fig. 9(b) y 9(d). Además, se observa la existencia de campo eléctrico en zonas alejadas de la línea de transmisión. De nuevo, la zona con campo es mayor para dos filas de mushrooms.

Si se comparan estas figuras con el comportamiento cuando tenemos una y dos filas de elementos 4CR, Figs. 10(c) y 10(d), podemos observar que la señal se atenúa pero el área con campo eléctrico es menor en el caso de $4 \mathrm{CR}$ que en el caso 

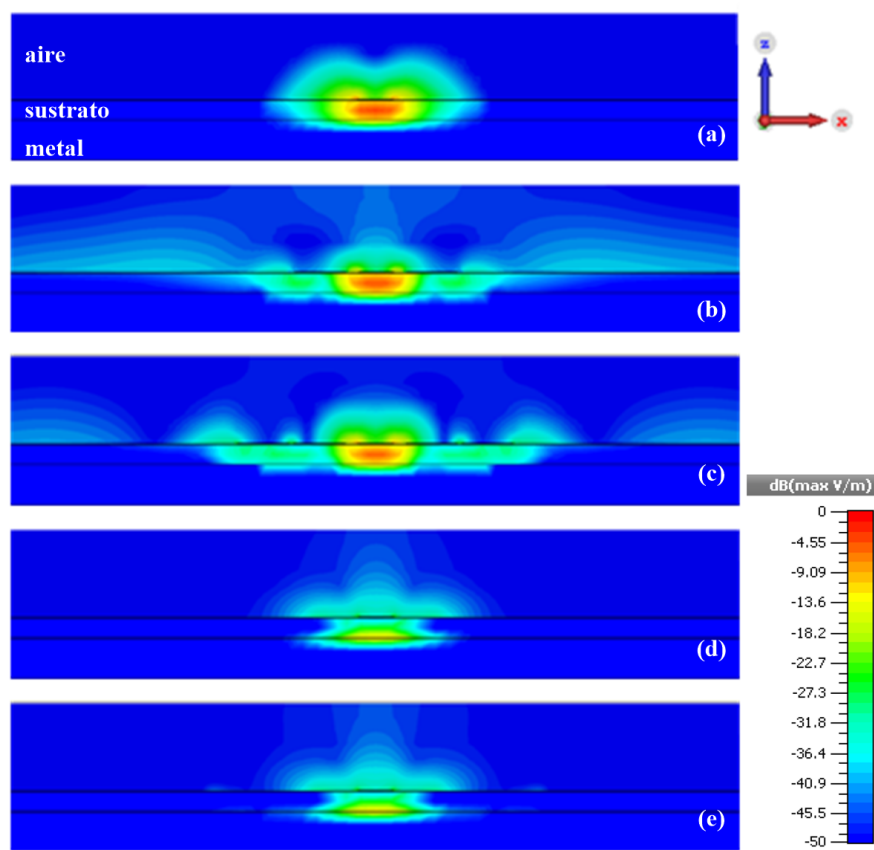

Fig. 11. Magnitud del campo eléctrico en el plano XZ $(y=30 \mathrm{~mm})$ : (a) línea de transmisión a $11.25 \mathrm{GHz}$, (b) línea con una fila de mushrooms a $11.5 \mathrm{GHz}$, (c) línea con dos filas de mushroom a $10.82 \mathrm{GHz}$, (d) línea con una fila de 4CR a $10.9 \mathrm{GHz}$, y (e) línea con dos filas de 4CR a $10.9 \mathrm{GHz}$.

de mushroom. Además, apenas hay diferencia en los resultados obtenidos con una y dos filas de 4CR.

También hemos estudiado la intensidad de campo eléctrico en la sección transversal de la línea de transmisión. La Fig. 11 muestra el campo en $y=30 \mathrm{~mm}$ a las frecuencias donde se produce la banda prohibida. Podemos observar que, en ausencia de inclusiones metálicas, los campos se concentran alrededor de la línea de transmisión Fig. 11(a). Sin embargo, cuando hay una fila de mushrooms a cada lado de la línea la distribución del campo eléctrico se extiende a una distancia mayor, Fig. 11(b). Lo mismo ocurre para dos filas de mushrooms a cada lado de la línea, Fig. 11(c). La inclusión de una fila de estructuras 4CR en cada lado de la línea produce una disminución del campo alrededor de ésta, Fig. 11(d), sin apreciarse cambios substanciales cuando se añade una fila más, Fig. 11(e). El campo eléctrico disminuye más rápido con la distancia, es decir, está más confinado que cuando tenemos mushrooms.

Por tanto, la inclusión de más de una fila de estructuras 4CR a cada lado de la línea de transmisión no produce variaciones significativas, y en consecuencia, la inclusión de una tercera o cuarta es innecesaria. Esta circunstancia no ocurre cuando se trata de estructuras mushroom. Por otro lado, las inserciones tipo manivela son estructuras más complejas y más difíciles de fabricar debido al mayor número de parámetros de diseño. Sin embargo, esta desventaja queda parcialmente compensada por su mejor comportamiento en frecuencia y por el menor número de elementos necesarios.

Desde un punto de vista práctico, la propuesta que en este trabajo se presenta destaca por la versatilidad geométrica de la inserción planteada, que es modificable a través de los numerosos parámetros constructivos del mismo (tanto los que constituyen la estructura $4 \mathrm{CR}$ como el posicionamiento rela- tivo con los elementos colindantes), lo que permite diferentes configuraciones del filtro.

\section{CONCLUSIONES}

En este trabajo se han analizado líneas de transmisión microstrip que incluyen elementos metálicos tridimensionales con distintas geometrías: mushroom y manivelas. Hemos encontrado que la inclusión de estructuras con simetría quiral produce un medio EBG con una banda prohibida de mayor anchura que cuando se utilizan mushrooms. Además, y a diferencia con lo que ocurre con mushrooms, la existencia de más de una fila de elementos $4 \mathrm{CR}$ no modifica significativamente la respuesta en frecuencia, dado que una única fila a cada lado de la línea de transmisión es suficiente. Se ha encontrado también un mayor confinamiento del campo eléctrico alrededor de la línea de transmisión en la banda prohibida, lo cual podría facilitar el aislamiento de elementos próximos a la línea.

Las nuevas estructuras propuestas basadas en inclusiones quirales constituyen un medio EBG, siendo de particular interés por presentar un gran ancho de banda de rechazo en la banda de estudio considerada en este trabajo.

\section{REFERENCIAS}

[1] Y. Rahmat-Samii, and H. Mosallaei, "Electromagnetic band-gap structures: classification, characterization, and applications," Eleventh Int. Conf. on Antennas and Propagation, IEE Conf. Publ., no. 480, vol. 2, pp. 560-564, 2001

[2] F. Capolino, "Theory and phenomena of metamaterials," CRC Press, Boca Raton, CRC Press, 2009.

[3] F. Jiang Liao, and L. Yang, "High impedance electromagnetic substrate and its application in plane traveling wave tubes," 2009 IEEE International Vacuum Electronics Conference, 2009.

[4] F. Yang, and Y. Rahmat-Samii, "Electromagnetic band gap structures in antenna engineering," in Cambridge University Press, Cambridge, UK, 2009.

[5] D. M. N. Elsheakh, et al.,'Antenna Designs with Electromagnetic Band Gap Structures, Metamaterial", InTech, pp. 403-473, 2012.

[6] F. Bilotti, et al., "On EBG structures for cellular phone applications,". AEU-International Journal of Electronics and Communications, 57(6), pp. 403-408, 2003.

[7] D. Sievenpiper, et al., "High-impedance electromagnetic surfaces with a forbidden frequency band," IEEE Transactions on Microwave Theory and Techniques, 47, (11), pp. 2059-2074, 1999.

[8] T. T. Nguyen, et al., "Design of a wideband mushroom-like electromagnetic bandgap structure with magneto-dielectric substrate," 6th International Conference on Information Technology and Applications, pp. 130-135, 2009.

[9] J. Lim, et al., "Enhanced broadband common-mode filter based on periodic electromagnetic bandgap structures," Microwave and Optical Technology Letters, 60, (12), 2932-2937, 2018.

[10] A. León, et al., "A new low-pass filter in asymmetrical finline topology using split-ring resonators," IEEE Latin America Transactions, 10(2), pp. 1445-1451, 2012.

[11] D. M. Pozar, "Microwave engineering," John Wiley \& Sons, 4th ed., pp. 147-152, 2012.

[12] A. J. García-Collado, et al., "Isotropic and homogeneous behavior of chiral media based on periodical inclusions of cranks," IEEE Microwave and Wireless Components Letters, 20, (3), pp. 175-177, 2010.

[13] A. J. García-Collado, et al., "Negative refraction of Chiral Metamaterial Based on Four Crank Resonators," Journal of Electromagnetic Waves and Applications, 26, (7), pp. 986-995, 2012.

[14] G. J. Molina-Cuberos, et al., "Electromagnetic activity of chiral media based on crank inclusions," IEEE Microwave and Wireless Components Letters, 19, (5), pp. 278-280, 2009. 


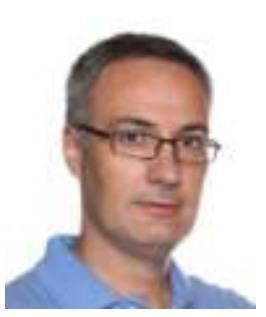

Ángel J. García-Collado was born in Murcia, Spain. He received the degree in Ingeniería de Telecomunicación from the Universidad Politécnica de Valencia, Spain, in 1998, and the Ph.D. degree in physics from the Universidad de Murcia in 2012. From 1998, works like researcher in the Universidad Católica de Murcia. From 2000 to 2003, he worked in different companies in the telecommunications sector. From the year 2003, devote exclusively to teaching practice and investigation in this University. Currently, he is part of the research group Teledetection, Geomatics and Applied Geographic Information Systems of this university. His current research activities include electromagnetic propagation in complex media and numerical methods in electromagnetism applied to chiral media.

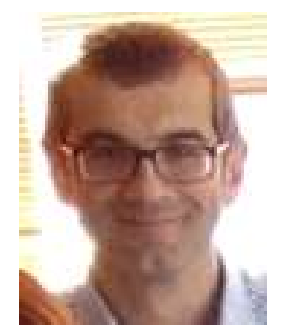

Gregorio J. Molina-Cuberos was born in Ronda (Málaga), Spain. He received the B.S. degree in applied physics from the Universidad de Granada, Spain, in 1994 and the MS and Ph.D. degrees in physics from the same university in 1998. From 1994 to 1999 he was at the Instituto Astrofísica Andalucía, in Granada, Spain, and from 1999 to 2001 at the Institut für Weltraumforschung in Graz, Austria. In 2001, he joined the Physics Department at Universidad de Murcia, where he became a Professor of Electromagnetism in 2008. His research interests are the electromagnetic characterization of planetary atmospheres and chiral media. He has been involved in several instruments onboard ESA and NASA space missions for solar system exploration.

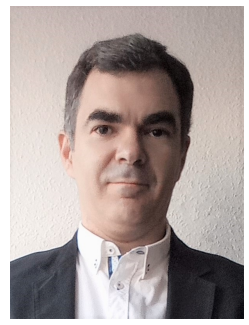

Óscar Fernández was born in Santander, Spain, in 1976. He received the Degree in telecommunications engineering and the Ph.D. degree from the University of Cantabria, Santander, in 2001 and 2007, respectively. He joined the Department of Communication Engineering, University of Cantabria, in 2001 where he became Assistant Professor of RF and Microwave in 2018. His current research interests include numerical methods in electromagnetism, chiral metamaterials and microwave measurements.

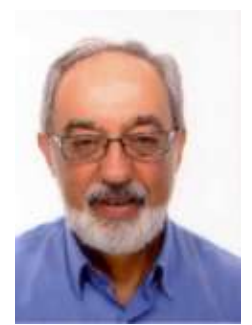

José Margineda was born in Balaguer (Lleida), Spain, in 1948. He received the degree in Physics from University of Madrid in 1972 and the Dr. in Science from University of Valladolid in 1977. From 1973 until 1979 he was with the Electricity and Electronics Department at University of Valladolid. In 1979, he joined the Physics Department at University of Murcia as an Assistant Professor, where he became a full Professor of Electromagnetism in 1983. He has been the head of the Physics Department from 1988 until 1996. In 2011, he moved to Electromagnetics and Electronic Department being head of Department until 2016. His scientific activity has been devoted mainly to electromagnetic measurements and instrumentation at microwave frequencies. At present, his current research interests are in chiral material characterization at microwave frequencies and their effects on wave propagation- $\mathrm{He}$ has been also interested in biological effects and food processing applications of microwaves. Prof Margineda is member of the IEEE from 1979, of European Physical Society from 1982 and of Real Sociedad Española de Física from 1974. 